\title{
Optimal Number of Pilots for OFDM Systems
}

\author{
Onésimo Ferreira ${ }^{1}$, Eduardo Rodrigues Vale ${ }^{2}$, Julio Cesar R. Dal Bello ${ }^{3}$ \\ ${ }^{1,2,3}$ (Telecommunications Department, School of Engineering / Federal Fluminense University, Brazil)
}

\begin{abstract}
The study addresses channel estimation by inserting pilots in the frequency domain, showing the performance of OFDM systems as a function of the number of pilots inserted. The channel estimation and performance analysis were done using the parameters BER (Bit Error Rate) and throughput, and characterized by the number of pilots inserted. Simulations show the influence on the BER by the number of inserted pilots and throughput performance. One of the most important applications for this study is in underwater acoustic communications, which works naturally with bands of lower frequencies. Thus, it is essential to use an adequate number of pilots in order to minimize the loss in the troughput of the system.
\end{abstract}

Key words: Channel Estimation, Fading, OFDM, Pilots, Throughput

\section{Introduction}

In a communication system, for the detection of signals transmitted, it becomes necessary to evaluate the behavior of the channel, so as to develop compensations leading to a lower error rate. One technique employed for this purpose consists of inserting pilots in the signal transmitted so that the receiver can, by use of estimation techniques, taking into consideration these effects of the channel.

Please note that, as a way of maintaining a high efficiency for the system, one should seek to maintain the number of pilots as low as possible, for a given error rate. This is of fundamental importance for the case of operation with underwater acoustic communication systems. In this case, the bandwidths are typically very small, which limits the use of a greater number of pilots.

Reference [1] adresses pilot distribution in a OFDM signal with reasonable depth, although the results were applied to a specific environment (i.e., mining).

Despite this paper is being directed to mobile communication systems, the results obtained have been applied successfully (although still at an experimental level) in the development of modems to be employed in underwater acoustic communication systems. We will be considering systems based on OFDM techniques, that is being used in cellular mobile systems of third and fourth generations.

To the knowledge of the authors, there is no specific technical literature that deals with the optimal choice of the distribution of the pilots in an OFDM ambient subject to multipath.

This paper is structured in the following form: item2 presents a brief description of the main subjects related to OFDM; item3 is directed toward signal detection; item 4 describes the system used for the simulations; item 5 relates to the throughput problem; item 6 presents the results of the simulations and item 7 gives the conclusion of this work.

\section{OFDM Considerations}

Orthogonal Frequency Division Multiplexing (OFDM) is a special form of Multicarrier Modulation, where a single data stream is transmitted through a number of subcarriers, at lower rate. OFDM can be viewed both as a modulation technique or a multiplexing technique.

OFDMA (Orthogonal Frequency Division Multiple Access) is a form to group signals from different sources, using OFDM technology. The main reason to use OFDM is to increase the robustness of the system against the frequency selective fading.

The spread of mobile radio signal can occur both in time and in frequency and is originated mainly by the phenomenon of multipath of the transmitted signal. Due to the different scatterers found in the environment between the mobile station and transmitter station, attenuated replicas of the transmitted signal arrive at the mobile station with different delays $(\mathrm{T})$, causing the time dispersion of the signal (this dispersion causes interference between symbols - ISI). This is characterized by parameters such as mean delay, delay spread $\left(\sigma_{\mathrm{T}}\right)$ and coherence bandwidth. This phenomenon is called frequency selective fading $[2,3]$.

The Doppler effect is due to the mobility of scatterers in the medium, and/or of the receiving station, associated with the variation of the arrival angles of multipath. As a consequence, there is a dispersion of the signal in the frequency, characterized by the following parameters: Doppler shift, Doppler spread and coherence time. This phenomenon is called time selective fading. This dispersion of the signal in the frequency causes interference between carriers.

The increased robustness against frequency selective fading occurs because in OFDM, the symbol rates of the subcarriers are low, thus the bandwidth of the transmitted signal (BW) is small. As the symbol period $\left(\mathrm{t}_{\mathrm{s}}\right)$ is 
the inverse of the frequency spacing $(\Delta \mathrm{f})$ and the frequency spacing is small, the symbol period will be long, allowing the insertion of a guard interval $\left(\Delta_{\mathrm{G}}\right)$, which is effective to avoid interference between symbols. Therefore, we have the validity of the following inequalities: $\Delta_{\mathrm{G}}>\mathrm{T}_{\max }$ and $\Delta_{\mathrm{G}}<\mathrm{t}_{\mathrm{s}} ; \mathrm{BW}<\mathrm{BW}_{\mathrm{C}} ; \mathrm{t}_{\mathrm{s}}>\sigma_{\mathrm{T}}$ and $\Delta \mathrm{f}$ $>\mathrm{BW}_{\mathrm{C}}$.

The robustness against the narrowband interference is due the orthogonality between the subcarriers. The high spectral efficiency occurs because the waveforms of the frequency spectrum are widely distributed and overlapping (due to the orthogonality) and therefore require less bandwidth.

The OFDM system is very effective in environments with ray multipath. With a single transmitter system (one carrier), fading or interference can cause a failure of all links but in a multicarrier system only a small percentage of subcarriers will be affected. An Error Correction Code can then be used to correct a few erroneous subcarriers [4].

To minimize the Inter Carrier Interference (ICI) problem, we can use the cyclic prefix technique, with $\Delta t>$ $\mathrm{T}_{\mathrm{C}}$ and ts $<\mathrm{T}_{\mathrm{C}}$, where $\Delta \mathrm{t}$ is the time between message retransmission. The techniques of guard intervals insertion and cyclic prefix can eliminate absolutely the interference among subcarriers.

The OFDM system can be easily generated using an IFFT (Inverse Fast Fourier Transform) and received through an FFT (Fast Fourier Transform) [5, 6, 7]. By employing rectangular FFT in the receiver, the interference between subcarriers can perfectly well be eliminated.

To generate an OFDM system successfully, the relation between all carriers must be carefully controlled to maintain the orthogonality between them. Therefore, the OFDM system is generated by firstly choosing the required spectrum, based on input data and the modulation scheme used. For each carrier that will be produced, some data is assigned for the transmission. The necessary bandwidth and carrier phase are calculated based on the modulation scheme (typically BPSK, QPSK or QAM). The required spectrum is then converted back to its signal in time domain using IFFT. The IFFT performs the transformation very efficiently, and provides a simple way to ensure the orthogonality of the carrier signals. The FFT transforms a cyclic signal in time domain in its equivalent frequency spectrum. This is done by finding the equivalent waveform, generated by a sum of orthogonal sinusoidal components. The amplitude and phase of sinusoidal components represent the frequency spectrum of the signal in time domain.

The IFFT performs the reverse process, transforming a spectrum (amplitude and phase of each component) into a signal in time domain.

The required orthogonal carriers for the OFDM signal can be generated easily by setting the amplitude and phase of each frequency, then running the IFFT. Since each data point in the frequency spectrum of an IFFT corresponds to the amplitude and phase of a set of orthogonal sinusoids, the reverse process ensures that the carriers generated are orthogonals [1].

\section{Channel Estimation and Pilot Allocation}

In order to detect the signal efficiently it is necessary to introduce some form of channel estimation.

Estimation methods based on pilots are those most commonly used, which are applicable in systems where the transmitter sends a known signal.

There are different possibilities for allocating pilots in time- frequency domain of an OFDM system [8]:

- A whole OFDM symbol can be allocated as a pilot (pilot allocation spaced in time and considering all frequencies).

- Pilots can be transmitted in individual subcarriers throughout the period of transmission (allocation of pilots spaced in frequency and considering full time).

The pilots can be allocated at intervals spaced in time and in frequency (allocation of pilots spaced in time and spaced in frequency).

\section{System Description}

In this item, it will be presented an OFDM model based on a platform developed to study the influence of pilot insertion.

The five main elements of the developed OFDM platform are: Symbol Source, OFDM Transmitter, Mobile Radio Channel, OFDM Receiver and Error Rate Meter.

The model consists basically of the following functions: the generation of the binary signal that will be transmitted, coding and decoding, modulation and demodulation, insertion and removal of pilots in the frequency domain, insertion and removal of stuffing zeros, use of FFT and IFFT, insertion and removal of cyclic prefix, simulation of Rayleigh multipath fading channel and the introduction of Gaussian noise (AWGN).

A total of 17 OFDM platforms were developed, each referring to different numbers of pilots $(2,3,4,5,6$, $7,11,13,16,21,26,31,51,61,76,101$ and 151). 
The Fig.1 represents an OFDM platform [9], developed by the authors, that utilizes pilot-based channel estimation method for equalization at the receiver side [4]. At the transmitter, binary data is mapped to a specific modulation (QPSK, 16QAM, 64QAM) and the modulated data undergoes serial-to-parallel (S/P) conversion, forming a vector of $\left(\mathrm{N}_{\mathrm{o}}-\mathrm{N}_{\text {pilot }}\right)$ symbols. $\mathrm{N}_{\mathrm{o}}$ is the number of occupied sub-carriers and $\mathrm{N}_{\text {pilot }}$ is the number of pilot sub-carriers. A number of $\mathrm{N}_{\text {pilot }}$ pilot symbols are then inserted into the modulated data, forming frequencydomain transmitted data $X_{k}$, of length $\mathrm{N}_{\mathrm{o}}$. $\mathrm{N}_{\mathrm{c}}$-point Inverse Fourier transform (IFFT) is performed on zero-padded $\mathrm{X}_{\mathrm{k}}$ to generate time-domain vector $\mathrm{x}(\mathrm{n})$. Cyclic prefix of $\mathrm{N}_{\mathrm{cp}}$ is then pre-appended to $\mathrm{x}(\mathrm{n})$ forming $\mathrm{X}_{\mathrm{f}}(\mathrm{n})$ vector of $\mathrm{N}_{\mathrm{c}}+\mathrm{N}_{\mathrm{cp}}$ symbols. After parallel to serial conversion (P/S), D/A conversion and low-pass filtering, $\mathrm{xf}(\mathrm{n})$ is transmitted over a linear time-invariant AWGN channel h(n) with noise $\mathrm{z}(\mathrm{n})$.

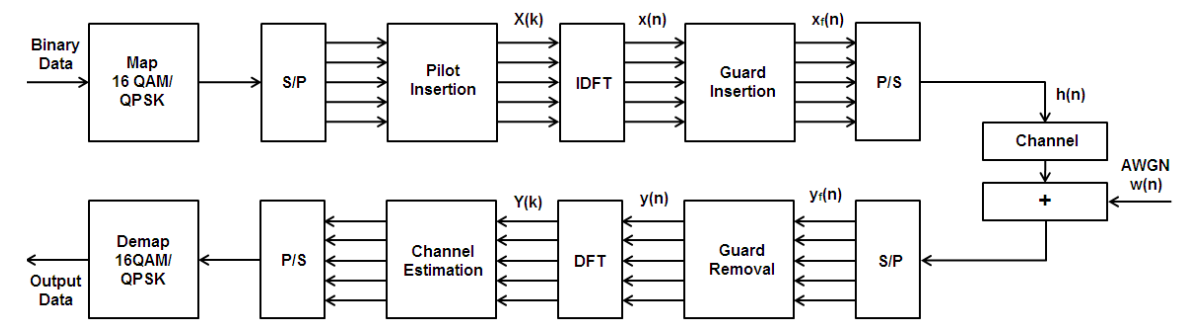

Figure 1: OFDM Platform

At the receiver, the opposite set of operations is performed. After D/A conversion and low-pass filtering, the received signal goes through a $\mathrm{S} / \mathrm{P}$ conversion and is stored in $\mathrm{y}_{\mathrm{f}}(\mathrm{n})$. Cyclic prefix is then removed from the $\mathrm{y}_{\mathrm{f}}(\mathrm{n})$, creating a $\mathrm{N}_{\mathrm{c}}$-dimensional received symbol vector $\mathrm{y}(\mathrm{n})$. $\mathrm{N}_{\mathrm{c}}$-point Fast Fourier Transform (FFT) is then applied on $y(n)$, creating frequency based $Y_{k}$ vector. Zero-padding is dropped from $Y_{k}$, shortening its length from $\mathrm{N}_{\mathrm{c}}$ to $\mathrm{N}_{\mathrm{o}}$. Equalization is applied using a pilot-based channel estimation method, and the pilot symbols are removed from the equalized signal. The equalized data then undergoes a $\mathrm{P} / \mathrm{S}$ conversion and demodulation, creating estimates of the transmitted binary data [10].

\section{Throughput Definitions}

The Throughput can be defined in the following ways:

\section{A. Throughput:}

Throughput $=(1-$ BER $) \times$ Data Bits Dados

B. Throughput (bits/subcarriers):

Throughput $=\underline{(1-\text { BER }) \times \text { Data Bits Dados }} \times \quad$ (Data Bits + Pilots Bits $)$

Therefore, we have: (Data Bits + Pilots Bits)

Throughput $=(1-$ BER $) \times$ Data Bits Dados

C. Effective Throughput (\%)

Throughput $=\left[(1-\right.$ BER $) \times\left(\right.$ Data Bits Dados $\left.\left.\times \mathrm{CM}_{\mathrm{I}}\right)\right]$

Where,

$\left[\left(\right.\right.$ Data Bits $\left.\times \mathrm{CM}_{\mathrm{I}}\right)+$ Pilots Bits $\left.)\right]$

$\mathrm{CM}_{\mathrm{I}}$ : Coding and Modulation Index $=$ Number of bits per symbol $=(1 / 2) * 4$

\section{Effective Throughput (bits/subcarriers)}

Throughput $=\left[(1-\right.$ BER $) \times\left(\right.$ Data Bits Dados $\left.\left.\times \mathrm{CM}_{\mathrm{I}}\right)\right] \times\left[\left(\right.\right.$ Data Bits $\left.\times \mathrm{CM}_{\mathrm{I}}\right)+$ Pilots Bits $\left.)\right]$

$$
\text { [(Data Bits } \left.\left.\times \mathrm{CM}_{\mathrm{I}}\right)+ \text { Pilots Bits) }\right]
$$

In the calculations and simulations it was considered the definition of the previous alternative $\underline{A}$.

\section{Simulation Results}

In the following sub-itens 6.1 to 6.6 , it is presented the:

- graphics of BER vs. Eb/No with the best curve fit,

- graphics of Throughput vs. Eb/No,

- results of BER and Throughput vs. Eb/No,

- graphics of BER vs. Pilots,

- graphics of Throughput vs. Pilots and

- results of BER and Throughput vs. Pilots. 


\subsection{BER vs. Eb / No with the best curve fit}

Fig.2 shows the curves and the values of the Error Rate Bit (BER) versus Eb/No, considering the following quantities of pilots: $2,3,4,5,6,7,11,13,16,21,26,31,51,61,76,101$ and 151.

It was considered the following values in decibels (dB) for Eb/No: 5, 6, 7, 8, 9, 10, 11, 12, 13, 14 and 15.

By analyzing the curve and the data separately for each pilot, it is concluded that the higher the Eb/No the lower will be the error rate. The curve shows that this gradual reduction of the error rate as a function of Eb/No is like an exponential decay.

Observe that the error rate decreases slowly for the following pilots: 2, 3, 4, 5, 6 and 7 . The error rate decreases slightly faster for the following pilots: $51,61,76,101$ and 151 . The error rate decreases with greater speed for the following pilots: 11, 13, 16, 21, 26 and 31 .

By analyzing the curve and data for all pilots, it is concluded that the error rate decreases for the following sequence of pilots: $5,3,2,4,6,7,61,101,76,51,151,16,26,11,13,31,21$.

The developer should consider the following number of pilots $16,26,11,13,31$ or 21 , and therefore the option with the lowest possible error rate is 21 pilots.

Once the focus of the investigation is the influence of the density of pilots in the performance of a OFDM system, it is concluded that the BER performance improves consistently with the inclusion of more pilots, but the throughput decreases due to the insertion of pilots.

For a small number of pilots inserted (2, 3, 4, 5, 6 and 7), the error rate is high. By increasing the number of pilots inserted (11, 13, 16, 21, 26 and 31), the error rate decreases. However, the results of simulation also show that if the number of pilots inserted $(51,61,76,101$ and 151) increase greatly, the system performance degrades, reaching intermediate values of BER.

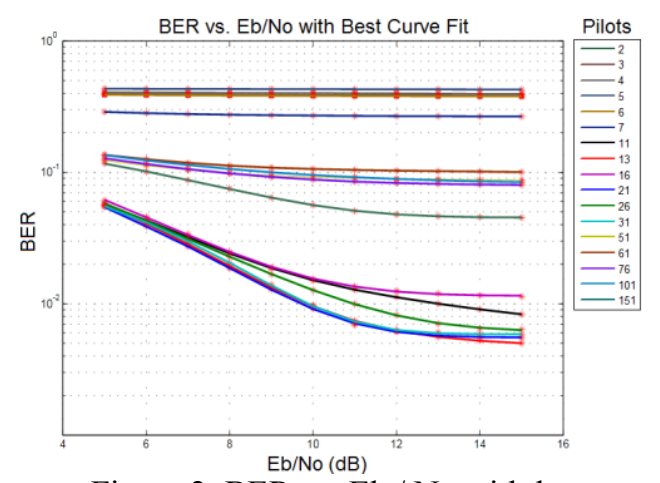

Figure 2. BER vs. Eb / No with best curve fit

\subsection{Throughput vs. Eb/No}

Fig. 3 show the curves and the values of throughput as a function of Eb/No, considering the following number of pilots: $2,3,4,5,6,7,11,13,16,21,26,31,51,61,76,101$ and 151.

It was considered the following values in decibels (dB) for Eb/No: 5, 6, 7, 8, 9, 10, 11, 12, 13, 14 and 15. By analyzing the curve and the data separately for each number of pilots, it is concluded that the higher the $\mathrm{Eb} /$ No the greater the throughput.

Fig. 3 shows the curves with a gradual increase of throughput as a function of Eb/No.Observe that the throughput grows slowly for the following number of pilots: $3,4,5,6$. The throughput grows a little faster for the following number of pilots: $7,51,61,76,101,151$. The throughput increases with greater speed for the following number of pilots: $11,13,16,21,26,31$.

Therefore, from Fig. 3 we can conclude also that, from a Eb/No equal to $13 \mathrm{~dB}$, the value of the throughput is constant, so the curves take the shape of a horizontal line.

By analyzing the curve and data for all pilots, it is concluded that the throughput decreases considering the following sequence of number of pilots: $11,13,16,21,26,31,51,61,76,7,101,6,4,151,2,3$, 5. With this result, it is concluded that, for working with greater throughput, the developer should consider the following quantities of pilots: $11,13,16,21,26$ or 31 , being 11 pilots the option with the highest throughput possible. 


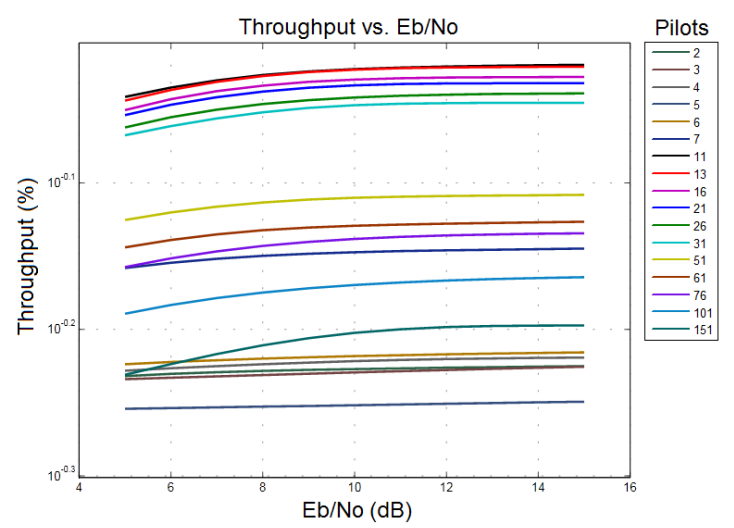

Figure 3. Throughput vs. Eb/No

\subsection{BER and Throughput vs. Eb/No}

Note that, when we have difficulties for retransmissions, the best possible error rate will be achieved with 21 pilots. However, this option does not represent the choice of higher throughput.

If we decide for the largest throughput possible, we should work with 11 pilots. This option does not present the lower error rate possible.

We can choose a middle ground, considering an average error rate and an average throughput. In this case it has the following alternative: to work with 13 pilots.

Although the option with 13 pilots presents a worse throughput than the option with 11 pilots and provide an error rate worse than the option with 21 pilots, it has a throughput better than the option with 21 pilots and has an error rate better than the option with 11 pilots.

\subsection{BER vs. Pilots}

It was concluded that, from an $\mathrm{Eb} / \mathrm{No}$ equal to $13 \mathrm{~dB}$, the value of the throughput is constant, so the curves take the form of a horizontal line.

As a result, following the discussions of this section, the error rate based on the number of pilots, assuming a fixed value of $\mathrm{Eb} / \mathrm{No}$, is equal to $13 \mathrm{~dB}$.

Fig.4 shows the curves and the values of the Error Rate (BER) versus the number of pilots, given Eb/No equal to $13 \mathrm{db}$. 151.

It was considered the following number of pilots: $2,3,4,5,6,7,11,13,16,21,26,31,51,61,76,101$ and

By analyzing the curve and the data, it is observed that the number of pilots 5, 2, 3, 4, 6, 7 have the highest rates of errors. The number of pilots $61,51,101,76,151$ show intermediate error rates and it is observed that for the number of pilots equal to $16,11,26,31,21$ and 13 , we have lower error rates.

By analyzing the curve and data for all pilots, it is concluded that the error rate decreases for the following sequence of pilots: $5,2,3,4,6,7,61,51,101,76,151,16,11,26,31,21,13$.

From these results it follows that given Eb/No equal to $13 \mathrm{~dB}$, for working with a lower error rate, the developer should consider the following quantities of pilots : 16, 11, 26, 31, 21 or 13, being 13 pilots the option with the lowest possible error rate.

\subsection{Throughput vs. Pilots}

In this section the throughput will be analyzed based on the number of pilots, considering a fixed value of $\mathrm{Eb} /$ No equal to $13 \mathrm{~dB}$.

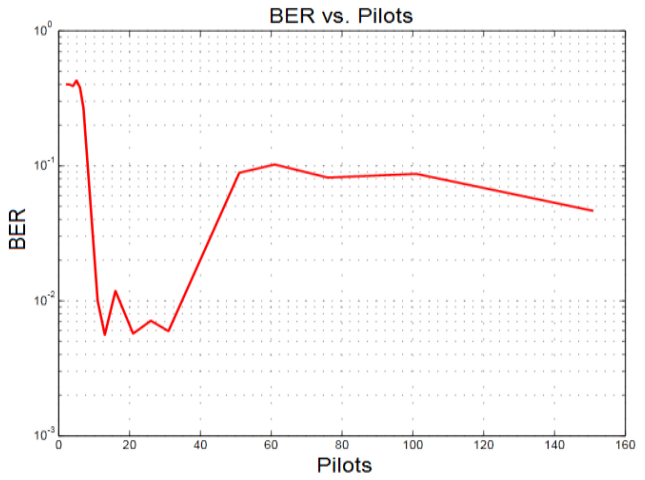

Figure 4. BER vs. Pilots 
Fig. 5 shows the curves and the values of throughput based on the number of pilots, given Eb/No equal to $13 \mathrm{~dB}$.

It was considered the following number of pilots: $2,3,4,5,6,7,11,13,16,21,26,31,51,61,76,101$ and 151.

By analyzing the curve and the data, it is observed that the number of pilots $11,13,16,21,26,31$ showed the largest throughput, the number of pilots 51,61, 76, 7, 101 have intermediate values of throughput and the number of pilots $151,6,4,2,3,5$ show the lowest throughput.

By analyzing the curve and data for all pilots, it is concluded that the throughput decreases for the following sequence of pilots: $11,13,16,21,26,31,51,61,76,7,101,151,6,4,2,3,5$. Therefore given Eb/No equal to $13 \mathrm{~dB}$, for working with greater throughput, the developer should consider the following number of pilots: $11,13,16,21,26$ or 31 , being 11 pilots the option with the highest throughput possible.

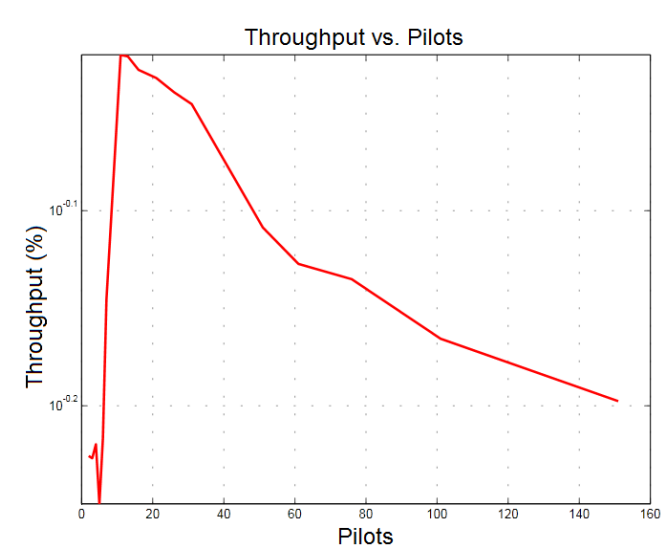

Figure 5. Throughput vs. Pilots

\subsection{BER and Throughput vs. Pilots}

If the developer decides for the lowest possible error rate, it should work with 13 pilots, but this option does not represent the choice of higher throughput. The alternative of working with a lower error rate is ideal for cases where there are difficulties in the retransmission.

If he decides for the higher throughput possible, it should work with 11 pilots, but this option does not present the lower error rate possible.

The developer may decide for a middle ground, i.e., it can be considered to work with the best error rate and an average throughput rate. In this case he has the following alternative: to work with 13 pilots.

The option with 13 pilots has the best error rate and a throughput rate slightly worse than the option with 11 pilots. On average, 13 pilots is the best option. Although the option with 11 pilots present the best throughput, on average, it is not recommended, as it provides an error rate far worse than the 13 pilots. Although the option with 21 pilots present an error rate much better than the option with 11 pilots, it presents a throughput worse than the options with 11 e 13 pilots. Again the option with 13 pilots is the best choice.

\section{Conclusion}

In this work the channel estimation and performance analysis were done using the parameters BER and throughput, and characterized by the number of pilots inserted. The results of exhaustive simulations performed made by an OFDM platform show the influence on the BER by the number of inserted pilots and throughput performance.

It was also presented the graphics BER vs. $\mathrm{Eb} / \mathrm{No}$, with the best curve fit, the graphics Throughput vs. $\mathrm{Eb} / \mathrm{No}$, the results BER and Throughput vs. Eb/No, the graphics BER vs. Pilots, the graphics Throughput vs. Pilots and the results BER and Throughput vs. Pilots.

By analyzing the curve BER vs. Eb/No, with the best curve fit, and the data for each pilot separately, it was concluded that the higher the $\mathrm{Eb} / \mathrm{No}$, the lower will be the error rate. The curve shows that the gradual reduction of error rate as a function of $\mathrm{Eb} / \mathrm{No}$, has an exponential decay.

By analyzing the curve Throughput vs. Eb/No and data from each pilot separately it is concluded that the higher the $\mathrm{Eb} / \mathrm{No}$, the greater the Throughput.

It was also concluded that from an $\mathrm{Eb} / \mathrm{No}$ equal to $13 \mathrm{~dB}$, the value of the throughput is constant, so the curves take the shape of a horizontal line.

If the developer chooses the smallest possible error rate, ideal for cases where there are difficulties in the data retransmission, it should work with 21 pilots. This option does not represent the choice of higher flow. 
If the developer decides for the largest flow possible, he should work with 11 pilots. This case does not represent the option of lower error rate.

Alternatively, the developer can choose a middle ground, here considered as an average error rate and an average throughput. In this case he has the alternative: to work with 13 pilots.

Thus, depending on the purpose for which the communications system is intended, one has various options applicable. Importantly, in the case of underwater acoustic communication systems we should opt for a smaller number of pilots, to avoid compromising the maximum available troughput.

\section{References}

[1] X. Yuan, Comb-Type Pilot-Aided OFDM Channel Estimation for Underground WLAN Communications, master thesis, CanadaQuebec, Université Laval, 2007.

[2] S. Hara and R. Prasad, Multicarrier Techniques for 4G Mobile Communications (Boston: Artech House,2003).

[3] N. Wang and S. D. Blostein, Adaptive Zero-Padding OFDM over Frequency-Selective Multipath Channels ( Canada: Hindawi Publishing Corporation, 2003).

[4] L. Litwin and M. Pugel, The Principles of OFDM (Indianapolis: 2001).

[5] R. van Nee and R. Prasad, OFDM for Wireless Multimedia Communications (Boston: Artech House, 2000).

[6] M. S. Akram, Pilot-based Channel Estimation in OFDM Systems (Copenhagen: Nokia Mobile Phones, 2007).

[7] H. Schulze and C. Lüders, Theory and Applications of OFDM and CDMA - Wideband Wireless Communications ( Germany: John Wiley \& Sons, 2005).

[8] M. S. Akram, Pilot-based Channel Estimation in OFDM Systems (Copenhagen: Nokia Mobile Phones, 2007).

[9] E. R. Vale and O. Ferreira, SIMULALTE - a LTE Simulator ( Registration Certificate of a Computer Program, Brazil, RS 11119-1, 2011).

[10] R. Prasad, OFDM for Wireless Communications Systems (Boston: Artech House, Universal Personal Communications Series, 2004). 